\title{
Surface chemistry modified upconversion nanoparticles as fluorescent sensor array for discrimination of foodborne pathogenic bacteria
}

\author{
Mingyuan Yin ${ }^{1}$, Chuang Jing ${ }^{1}$, Haijie Li ${ }^{1}$, Qiliang Deng ${ }^{1 *}$ and Shuo Wang ${ }^{1,2^{*}}$
}

\begin{abstract}
Background: The identification of foodborne pathogenic bacteria types plays a crucial role in food safety and public health. In consideration of long culturing times, tedious operations and the desired specific recognition elements in conventional methods, the alternative fluorescent sensor arrays can offer a high-effective approach in bacterial identification by using multiple cross-reactive receptors. Herein, we achieve this goal by constructing an upconversion fluorescent sensor array based on anti-stokes luminogens featuring a series of functional lanthanide-doped upconversion nanoparticles (UCNPs) with phenylboronic acid, phosphate groups, or imidazole ionic liquid. The prevalent spotlight effect of microorganism and the electrostatic interaction between UCNPs and bacteria endow such sensor array an excellent discrimination property.
\end{abstract}

Results: Seven common foodborne pathogenic bacteria including two Gram-positive bacteria (Staphylococcus aureus and Listeria monocytogenes) and five Gram-negative bacteria (Escherichia coli, Salmonella, Cronobacter sakazakii, Shigella flexneri and Vibrio parahaemolyticus) are precisely identified with 100\% accuracy via linear discriminant analysis (LDA). Furthermore, blends of bacteria have been identified accurately. Bacteria in real samples (tap water, milk and beef) have been effectively discriminated with $92.1 \%$ accuracy.

Conclusions: Current fluorescence sensor array is a powerful tool for high-throughput bacteria identification, which overcomes the time-consuming bacteria culture and heavy dependence of specific recognition elements. The high efficiency of whole bacterial cell detection and the discrimination capability of life and death bacteria can brighten the application of fluorescence sensor array.

Keywords: Upconversion nanoparticles, Foodborne bacteria, Sensor array, Identification

\section{Background}

The detection and discrimination of foodborne pathogenic bacteria is a crucial issue in environmental monitoring, food safety and early diagnosis of diseases [1-5].

\footnotetext{
*Correspondence: yhdql@tust.edu.cn; wangshuo@nankai.edu.cn 1 State Key Laboratory of Food Nutrition and Safety, School of Food Engineering and Biotechnology, College of Chemical Engineering and Materials Science, Tianjin University of Science and Technology, Tianjin 300457, People's Republic of China

Full list of author information is available at the end of the article
}

Every year over 300 million illnesses and more than 5 million deaths result from pathogenic bacterial infection, causing inestimable loss of property [6]. Therefore, the constant threats from existing and emerging foodborne pathogenic bacteria make the rapid and reliable identification and quantification of bacterial species an important index for shriveling foodborne pathogenic bacteria contamination $[3,7]$.

Up to present, the extensively adopted methods for bacterial identification include plate cultivation,

(c) The Author(s) 2020. This article is licensed under a Creative Commons Attribution 4.0 International License, which permits use, sharing, adaptation, distribution and reproduction in any medium or format, as long as you give appropriate credit to the original author(s) and the source, provide a link to the Creative Commons licence, and indicate if changes were made. The images or other third party material in this article are included in the article's Creative Commons licence, unless indicated otherwise in a credit line to the material. If material is not included in the article's Creative Commons licence and your intended use is not permitted by statutory regulation or exceeds the permitted use, you will need to obtain permission directly from the copyright holder. To view a copy of this licence, visit http://creativeco mmons.org/licenses/by/4.0/. The Creative Commons Public Domain Dedication waiver (http://creativecommons.org/publicdomain/ zero/1.0/) applies to the data made available in this article, unless otherwise stated in a credit line to the data. 
morphological structure observation, gene and immunological characteristics analysis $[4,6]$. However, the traditional bacterial species identification methods heavily rely on time-consuming and low-accuracy phenotypic characterizations such as serotype by Gram staining and biochemical methods [4]. Polymerase chain reaction (PCR), gene sequencing, surface enhanced Raman scattering (SERS), and mass spectrometry as well as a series of specificity sensing methods for analysis bacteria based on the affinity reagents (including peptide, phage, antibiotic, antibody, and aptamer) have been widely employed to precise traceability analysis [8-13], the main limitations of these methods are highly reliant on expensive reagents, sophisticated costly and bulky equipment, elusive manipulations, and trained personnel and handling conditions [7, 13]. Furthermore, to ensure effective treatments, timely and reliable diagnosis of pathogen infection is the primary step [14]. Lacking of reliable and punctual pathogenic contamination information, the suboptimal selective drug treatment can delay the best treatment timing and lead to strains undergo mutations and acquire antibiotic resistance $[6,12]$. A dire prediction shows that 10 million people can be killed by antibioticresistant bacteria infections worldwide by 2050 [6]. Thus, developing timely and efficient methods to discern foodborne pathogenic bacteria are urgent need for food security and public health $[7,12,13]$.

The fluorescent probe is an alternative and promising tool for the identification of pathogenic bacteria with great temporal and spatial sampling capability, rapid response, and high sensitivity and simplicity [15-18]. The biosensor for pathogenic bacteria identification based on fluorescent responses has received more and more attention [16, 19]. However, conventional luminescence elements generally convert high-energy photons into low-energy photons (such as quantum dot, fluorescent conjugated polymer, and fluorochrome) [20], and the photobleaching and background noise as well as ground substance disturbance are still inescapable [6]. This has led to their narrow scope of application, low level working concentration and weak labeling degrees to analyte, generating compromised sensitivity and accuracy of identification [6]. To tackle these issues, the fluorescence probes for bacterial identification based on the conventional sensing elements will be thoroughly covered by lanthanide-doped upconversion nanoparticles (UCNPs) materials with anti-stokes luminescence [10-12, 21].

Diametrically opposed to the conventional luminescence sensing elements, UCNPs materials are able to convert low-energy photons (such as near-infrared light) into high-energy photons (such as visible and ultraviolet light), which is ideal for sensing analyte in complex environment [22-24]. Moreover, UCNPs-based probes have excellent photo-stability, multicolor tunable property, less toxic elements, negligible autofluorescence background and the minimal photo blinking and photo bleaching $[10-12,21,25]$, which can heavily improve the sensitivity and reliability of detection. In addition, surface chemistry modification further endows UCNPs materials versatile properties and enables these functional UCNPs materials to realize the tunable charges and solubility, and target diversity, which provides a large library for sensor selection [10, 12, 22, 26, 27].

Recently, the fluorescent sensor arrays constructed from a series of functioned UCNPs probes have exhibited excellent power for identification of analytes (such as phosphate compounds, grape wines, proteins, glycated hemoglobin, vitamin B12, viruses, biotinylated antibodies and human IgG and IgM antibodies) with high classification accuracy in a timely and cost-effective manner [22, 28-33]. UCNPs materials have been widely utilized to realize the quantification of single bacteria in previous researches [9-12, 34]. In addition, multicolor upconversion nanoparticles modified with specific recognition element (aptamers and antibody) have also been constructed for the detection of multiplex pathogenic bacteria $[35,36]$.

In this research, we introduce the multivalent interactions between UCNPs materials and bacteria to improve the fluorescence response towards bacteria, taking advantage of the prevalent spotlight effect of microorganism as well as the electrostatic interaction $[10,12]$. Three surface chemistry modification UCNPs probes (bearing one positively charged imidazole ionic liquid groups, two negatively charged phenylboronic acid or phosphate groups) are designed and synthesized. A fluorescent sensor array composed of the three UCNPs probes is constructed, which can successfully distinguish seven representative foodborne pathogenic bacteria (Escherichia coli (E. coli), Staphylococcus aureus (S. aureus), Salmonella, Listeria monocytogenes (L. monocytogenes), Cronobacter sakazakii (C. sakazakii), Shigella flexneri (S. flexneri) and Vibrio parahaemolyticus (V. parahaemolyticus)) through pattern recognition with linear discriminant analysis (LDA) and realize the bacterial analyses from real samples (tap water, milk and beef).

\section{Materials and methods Materials}

Lanthanide acetate hydrates $\left(99.9 \%, \mathrm{Ln}(\mathrm{Ac})_{3}, \mathrm{Ln}=\mathrm{Y}, \mathrm{Yb}\right.$, and Er) were obtained from Alfa Aesar Co. Ltd. (Ward Hill, MA, USA). Oleic acid (OA, 90\%), 1-octadecene (ODE, 90\%), tetraethyl orthosilicate (TEOS, 98\%), allyltriethoxysilane (ATS, 97\%), 1-vinylimidazole (VID, 99\%) and 1-bromooctane (99\%) were obtained from J\&K Chemical (Beijing, China). Vinylphosphonic acid (VPA, 
97\%) and 4-vinylphenylboronic acid (VPBA, 95\%) were purchased from Sigma Aldrich (St. Louis, MO, USA). Triton X-100 was supplied by GFCO Chemical (Hongkong, China). Azodiisobutyronitrile (AIBN, 98\%), ethanol (95\%), cyclohexane (95\%) and ammonia solution (25\%) were brought from North Tianyi Chemical Reagent Factory (Tianjin, China). Luria-bertani (LB) broth, agar powder, nutrient agar/broth, brain heart infusion broth, dimethyl sulfoxide (DMSO) and phosphate buffer saline (PBS) were ordered from Beijing Solarbio Science \& Technology Co., Ltd. (Beijing, China). Double distilled water (DDW, $18.2 \mathrm{M} \Omega \mathrm{cm}^{-1}$ ) was produced by a Water Pro water purification system. E. coli (ATCC25922), S. aureus (ATCC25923), Salmonella (CICC10867), L. monocytogenes (ATCC7644), C. sakazakii (ATCCBAA894), S. flexneri (ATCC12022) and V. parahaemolyticus (ATCC17802) were ordered from BeNa Culture Collection Co., Ltd. (Beijing, China). Milk and beef were purchased from the local supermarket. Tap water was collected from water pipe in Tianjin University of Science and Technology campus. Other reagents were at least of analytical grade and without further purification.

\section{Characterization}

Fluorescence spectra were measured on an F-7000 fluorescence spectrometer (Hitachi, Tokyo, Japan) attached with an external $980 \mathrm{~nm}$ laser (2 W, continuous wave with $1 \mathrm{~m}$ fiber, Beijing, China) instead of internal excitation source. Transmission electron microscopy (TEM, $200 \mathrm{kV}$ ) images were obtained on a JEOL 2010F (JEOL, Japan) with an attached energy-dispersive X-ray spectroscope (EDS). Fourier transform infrared (FT-IR) spectra $\left(4000-400 \mathrm{~cm}^{-1}\right)$ in $\mathrm{KBr}$ were recorded in a Vector 22 FT-IR spectrophotometer (Bruker, Karlsruhe, Germany). The zeta potentials of UCNPs materials were measured at room temperature in neutral water solution with a Zetasizer Nano ZS90 (Malvern, Worcestershire, U.K.). Ultraviolet-Visible (UV-Vis) absorbance spectra were recorded on a Shimadzu UV-2700 UV-Vis spectrophotometer (Shimadzu, Japan). The suspension of nanoparticles was prepared using an ultrasonic bath SBL-10DT (Ningbo, China). Thermogravimetric analysis (TGA) was carried out by STA 449 F5 Jupiter Netzsch thermogravimeter (Netzsch, Selb, Germany).

\section{Synthesis of UCNPs@COPs materials}

The oil-solvent lanthanide-doped UCNPs and the copolymers (COPs) were first prepared as procedure described in Additional file 1. The UCNPs@COPs materials were obtained by inverse microemulsion method as following, UCNPs $(0.04 \mathrm{mM})$ were dissolved into acetonitrile $(6.0 \mathrm{~mL})$ containing Triton X-100 $(0.1 \mathrm{~mL})$ under stirring. Next, ammonia solution $(0.08 \mathrm{~mL})$ and
Triton X-100 $(0.4 \mathrm{~mL})$ were added and sonicated for $20 \mathrm{~min}$. Subsequently, TEOS $(40 \mu \mathrm{L})$ and the as-prepared COPs $(0.04 \mathrm{mM})$ were slowly dropped in sequence. After the system was stirred at room temperature for $24 \mathrm{~h}$, UCNPs@COPs were collected and washed with ethanol for several times.

\section{Bacteria culturing and counting}

Seven representative foodborne pathogenic bacteria including E. coli, S. aureus, Salmonella, L. monocytogenes, C. sakazakii, S. flexneri and V. parahaemolyticus were obtained as previous process [12]. The obtained bacteria were centrifuged at $3000 \mathrm{~g}$ for $5 \mathrm{~min}$, washed with physiological saline solution for three times, and diluted to the desired concentration. The number of bacteria $\left(\mathrm{OD}_{600}=0.1\right)$ were estimated by the plate counting method (Additional file 1: Table S2). The death E. coli and $S$. aureus were obtained by ultrasonic treatment. Tubes containing $1 \mathrm{~mL}$ aliquots of bacteria suspension $\left(\mathrm{OD}_{600}=0.5\right)$ were treated by ultrasonic for $20 \mathrm{~min}$ (The ultrasonic operates at a nominal frequency of $40 \mathrm{kHz}$ ).

\section{Optimization of sensor array working conditions}

In order to obtain the optimal detection conditions, $E$. coli $\left(\mathrm{OD}_{600}=0.5\right)$ was chosen as a model bacterium. The fluorescence spectra of the different concentrations of the three UCNPs@COPs materials $(0.05,0.10,0.15$, $0.20,0.25$, and $0.30 \mathrm{mg} \mathrm{mL}^{-1}$ ) treated with E. coli were recorded. The optimum incubating time of the three UCNPs@COPs materials treated with E. coli was investigated by measuring the fluorescence emission intensity at certain intervals $(2,4,6,10,20,25,30,35$, and $60 \mathrm{~min})$.

\section{Bacterial identification based on sensor array}

Generally, the solutions of the three UCNPs@COPs $\left(0.20 \mathrm{mg} \mathrm{mL} \mathrm{m}^{-1}\right)$ were prepared in physiological saline with 10\% DMSO, and mixed with bacterial solutions $(\mathrm{v} / \mathrm{v}=1: 1)$. Subsequently, the fluorescence spectra of the mixtures were recorded. The fluorescence emission intensity at $550 \mathrm{~nm}$ of each UCNPs@COPs material for each bacterial species was collected, and the training set was obtained by selecting six independent replicates.

\section{Fluorescence response patterns}

The collected date of fluorescence response patterns $\left(\left(\mathrm{F}_{\mathrm{i}}-\mathrm{F}_{0}\right) / \mathrm{F}_{0}\right)$ were analyzed using SPSS (IBM SPSS Statistics 22), and the raw data matrix $(3 \times \mathrm{UCNPs@COPs} \times 7$ bacteria $\times 6$ replicates) was processed using the linear discriminant analysis (LDA) and hierarchical clustering analysis (HCA). For classification, the training set was used to perform LDA, obtaining the corresponding scores as well as coefficients. For HCA, the average linkage method was adapted. 


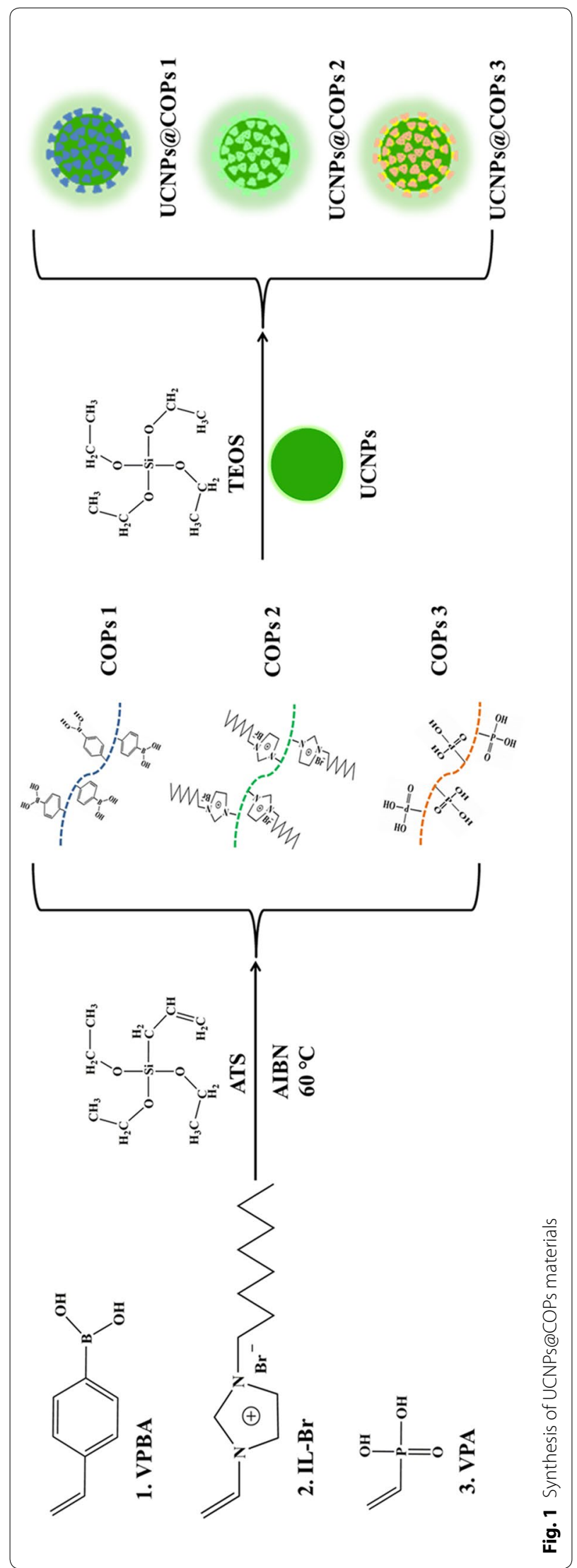



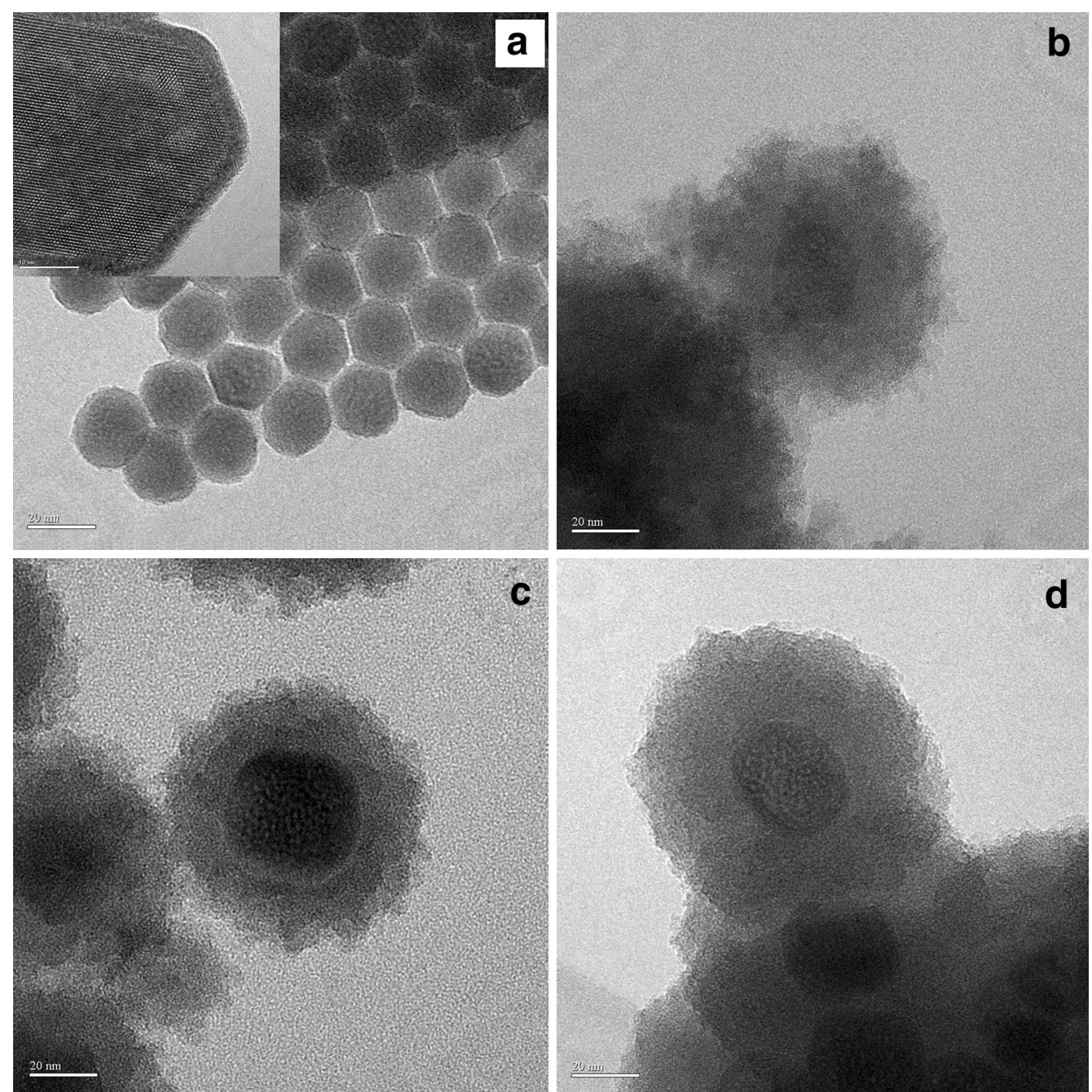

Fig. 2 TEM images of UCNPs (a), UCNPs@COPs 1 (b), UCNPs@COPs 2 (c) and UCNPs@COPs 3 (d)

\section{Identification of bacteria in real sample}

As common sources of bacterial contamination, tap water, milk and ground beef were selected as real samples. Standard addition method with a series of pretreatment procedure was performed referencing to the previous report [36]. For the tap water (following the sanitary standards for drinking water quality of the state standard of the People's Republic of China (GB 57492006) [37]), sample (100.0 mL) was collected from water pipe without any pre-treatment and added the desired concentration standard bacterial stock for the following detection. Specifically, for the milk sample (about $6 \%$ protein content, $7 \%$ fat content and $15 \%$ calcium content), the desired concentration standard bacterial stock was added to $25.0 \mathrm{~mL}$ of aseptic sample. After centrifugation at $7000 \mathrm{~g}$ for $10 \mathrm{~min}$ to remove the upper layer of cream, the milk sample was then diluted with sterile water (1:9). For the ground beef sample (about $85 \%$ lean content and $15 \%$ fat content), sample ( $25.0 \mathrm{~g})$ were added the desired concentration of standard bacterial stock and homogenized for $5 \mathrm{~min}$ in $225.0 \mathrm{~mL}$ of aseptic $1 \times$ PBS. Then, the solution was maintained for $30 \mathrm{~min}$ to precipitate macroaggregates and seston. Finally, the supernatant was collected through a $0.45 \mu \mathrm{m}$ filtration membrane. After these pre-treatment steps, both the spiked samples and raw samples (as control) were subjected to UCNP@ COPs probes following the general procedure. And the total acquisition time of the bacterial detection in different samples was estimated to $15 \mathrm{~min}$ (tap water), $30 \mathrm{~min}$ (milk), and $60 \mathrm{~min}$ (beef). 


\section{Results and discussion}

\section{Characterization of UCNPs materials}

In order to develop UCNPs-based materials for bacteria recognition, three surface chemistry modification versatile UCNPs materials with functional groups including phenylboronic acid (UCNPs@COPs 1), imidazole ionic liquid (UCNPs@COPs 2), and phosphate groups (UCNPs@COPs 3) were designed as sensing probes. As shown in the Fig. 1 and Additional file 1: Table S1, allyltriethoxysilane (ATS) and functional monomers (4-vinylphenylboronic acid (VPBA), 1-octyl-3-vinylimidazolium bromide ionic liquid (IL-Br) and vinylphosphonic acid (VPA)) were employed to pre-compose cross-linked COPs via free-radical polymerization triggered by AIBN, then UCNPs@COPs materials were obtained by grafting COPs onto the surface of UCNPs via inverse microemulsion method.

The elemental analyses were first carried out by EDS to confirm the composition of UCNPs materials (Additional file 1: Figure S1). Common elementals of $\mathrm{F}, \mathrm{Na}, \mathrm{Y}, \mathrm{Yb}$, and Er were observed in all the obtained UCNPs@COPs materials. Additionally, the characteristic elementals such as B in UCNPs@COPs 1, N in UCNPs@COPs 2, and P in UCNPs@COPs 3 were emerged, respectively, indicating that COPs were successfully grafted onto UCNPs.

The investigation of TEM images exhibited that monodisperse hexagonal nanoprisms of UCNPs with a uniform particle size about $20 \mathrm{~nm}$ was formed, and the COPs layers (around $20 \mathrm{~nm}$ thickness) have been coated onto the surface of UCNPs (Fig. 2). Although the morphologies of the three UCNPs@COPs materials exhibited discrepant core-shell structure, it was not studied in detail because the reshaping did not effect on the fluorescence properties. XRD analysis (Additional file 1: Figure S2) revealed that the position and peak shape of major diffraction peaks of UCNPs@COPs in $2 \theta$ were good consistent with the bare UCNPs and the standard alignment card (JCPDS standard card number 16-0334), indicating that all the materials were equipped with $\beta$-phase crystal. And no impurity diffraction peak was found in UCNPs@ COPs, indicating that the hexagonal-phase structure was not disturbed by the COPs layers.

Compared with FITR spectra of UCNPs, the most characteristic bands of UCNPs@COPs materials revealed significant changes in the band intensity and vibration frequency (Additional file 1: Figure S3). The characteristic peaks of UCNPs at 1419 and $1560 \mathrm{~cm}^{-1}$ were attributed to asymmetric and symmetrical stretching vibration peaks of carboxyl groups of OA molecules, and the obvious peaks at 2854, and $2926 \mathrm{~cm}^{-1}$ raised from the stretching vibration peak of the methyl and methylene groups of OA molecule. Grafting COPs on the surface of UCNPs, the characteristic peaks of OA molecule diminished or disappeared. Meanwhile, the stretching vibration characteristic peaks of $\mathrm{Si}-\mathrm{O}-\mathrm{Si}$ were observed at 1085,1087 , and $1091 \mathrm{~cm}^{-1}$ in the three UCNPs@ COPs materials. In addition, the characteristic peaks at 1387 and $1636 \mathrm{~cm}^{-1}$ belonged to the stretching vibration peaks of B-O and benzene ring structure of UCNPs@ COPs 1 . The characteristic peaks at 1504 and $1633 \mathrm{~cm}^{-1}$ raised from the stretching vibration of imidazole ring structure of UCNPs@COPs 2. The characteristic peaks at 1389 and $1627 \mathrm{~cm}^{-1}$ were attributed to the stretching vibration peaks of $\mathrm{P}=\mathrm{O}$ and $\mathrm{P}-\mathrm{O}$ of UCNPs@COPs 3. Triton $\mathrm{X}-100$ was involved during the preparation of the materials. Therefore, the removal of such compound on the particle surface was investigated via the monitoring of UV-Vis absorbance spectra. As shown in Additional file 1: Figure S4A, the key ultraviolet absorbance peaks of triton X-100 were significantly observed in the pre-treated UCNPs@COPs, however, disappeared in the post-treated UCNPs@COPs. In addition, we also checked Triton X-100 in the washing solution. As shown in Additional file 1: Figure S4B, the concentration of Triton $\mathrm{X}-100$ reduced as the washing times increase. Consistent with previous study [38], all these results indicated that Triton X-100 was removed from UNCPs materials via washing with ethanol.

Thermal stabilities of the three UCNPs@COPs materials were further characterized by the thermogravimetric analysis (TGA). As shown in Additional file 1: Figure S5, the first weight loss of the three UCNPs@COPs materials occurred around $100{ }^{\circ} \mathrm{C}$, which was arisen from the desorption of atmospheric moisture. The second thermal decomposition observed in the range of $160-400{ }^{\circ} \mathrm{C}$ was arisen from the dehydrations of UCNPs@COPs materials. Final thermal decomposition (in the range from 400 to $650{ }^{\circ} \mathrm{C}$ ) was attributed to the decomposition of the COPs layers. From 650 to $800{ }^{\circ} \mathrm{C}$, thermal decomposition became neglected, and the weight losses of UCNPs@ COPs 1, 2, and 3 were steady decline to $17 \%, 19 \%$ and $16 \%$, respectively, which were more than that of the bare UCNPs (11\%). TGA results revealed the good thermal stabilization of UCNPs@COPs materials.

Zeta potential is a significant factor for fluorescence probe, and the three UCNPs@COPs materials revealed significant difference in terms of surface potentials. As shown in Additional file 1: Figure S6, the bare UCNPs displayed the positive surface potentials $(+3.85 \mathrm{mV})$, and the surface potentials of UCNPs@ COPs 1, UCNPs@COPs 2, and UCNPs@COPs 3 were $-17.13 \mathrm{mV},+31.93 \mathrm{mV}$, and $-30.63 \mathrm{mV}$, respectively. All these results further confirmed that the COPs have been successfully modified onto the surface of UCNPs materials. 


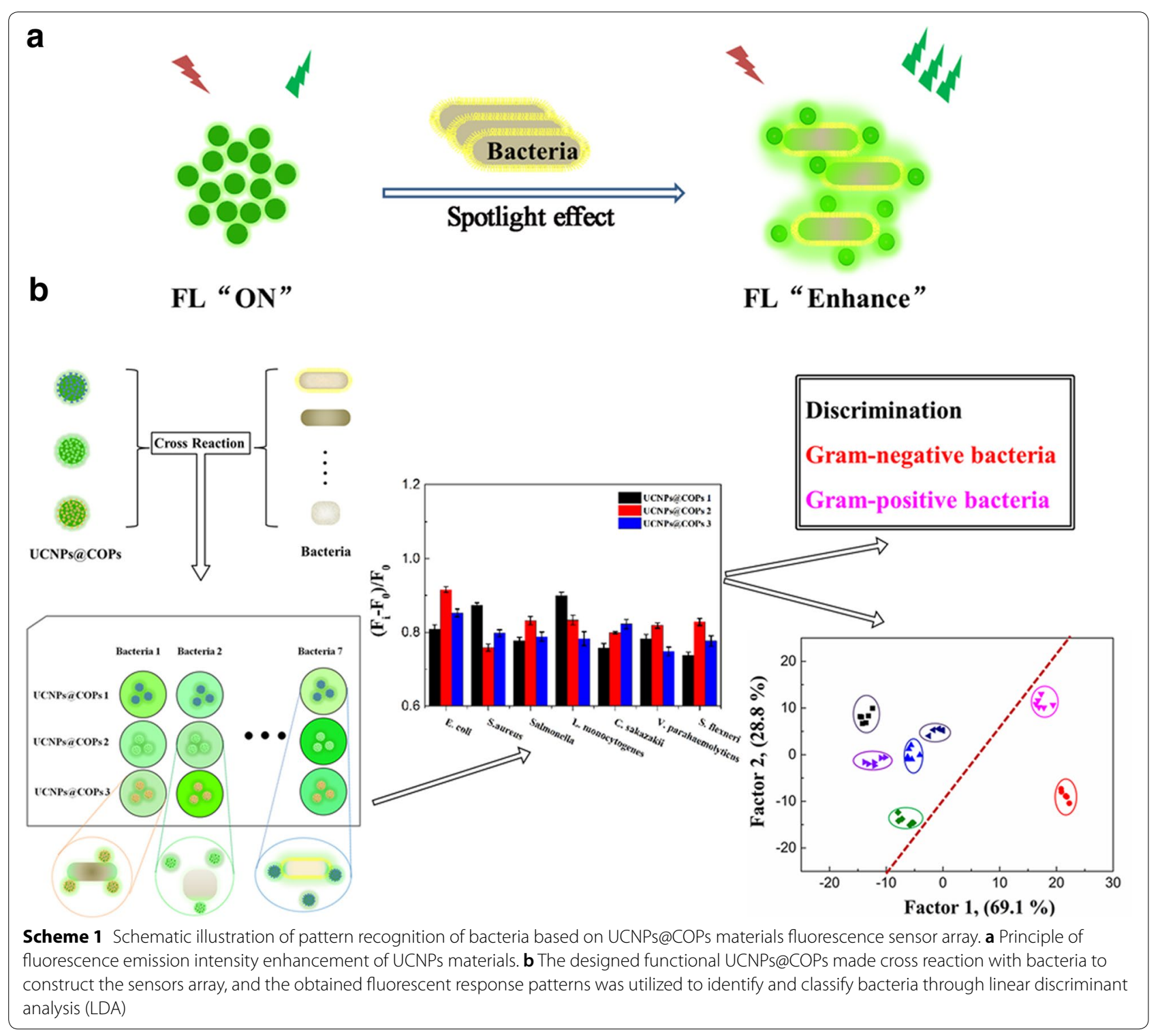

As an excellent anti-stokes luminescent element, the fluorescence emission spectra of the three UCNPs@ COPs materials under $980 \mathrm{~nm}$ excitation wavelengths were further investigated in physiological saline solution/ DMSO (9:1). As expected, the prominent fluorescence emission spectra of the three UCNPs@COPs materials showed the typical green upconversion emissions of $\mathrm{NaYF}_{4}: \mathrm{Er}^{3+}, \mathrm{Yb}^{3+}$ (the characteristic of $\mathrm{Er}^{3+}$ corresponded to the $\left({ }^{2} \mathrm{H} 11 / 2\right.$ and $\left.{ }^{4} \mathrm{~S} 3 / 2\right) \rightarrow{ }^{4} \mathrm{I} 15 / 2$ transitions $)$ [27], and the fluorescence emission intensity of the three UCNPs@COPs materials decreased slighter than that of the bare UCNPs at the same concentration, which was attributed to the weakened luminescence caused by the capping agents COPs (Additional file 1: Figure S7A). Meanwhile, the capping agents COPs with different functional groups did give rise to certain difference of fluorescence emission intensity. Although the fluorescence emission intensities of UCNPs@COPs probes were weaker than that of the bare UCNPs materials, the fluorescence responses of all the three UCNPs@COPs probes caused by the alive E.coli were more sensitivity than that of the bare UCNPs, which demonstrated the COPs layer had a crucial role for the bacteria binding (Additional file 1: Figure S7B). All above results have demonstrated that the three UCNPs@COPs materials not only inherited the good luminescence properties of UCNPs materials, but also gave abundant action sites, which could be ideal materials in the sensing field. 

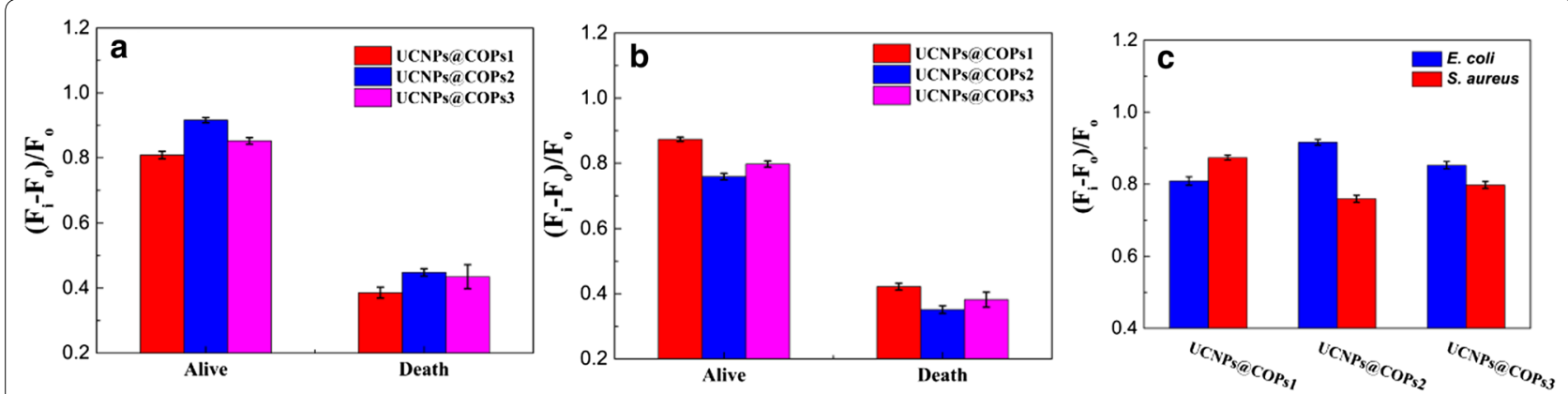

Fig. 3 a Fluorescent response of UCNPs@COPs probes treated with alive and death E. coli $\left(\mathrm{OD}_{600}=0.5\right)$. b Fluorescent response of UCNPs@COPs probes treated with alive and death S. aureus $\left(\mathrm{OD}_{600}=0.5\right)$. c Fluorescent response of UCNPs@COPs probes treated with alive E. coli and S. aureus $\left(\mathrm{OD}_{600}=0.5\right)$

\section{Role of UCNPs@COPs materials}

Generally, the bacterial cell can improve the fluorescence emission of UCNPs material due to the spotlight effect of cell and the interaction [10-12]. The three UCNPs@ COPs probes with different functional groups can further reinforce the interaction and exhibit the different fluorescence signal response. The strongly positive charged imidazole ionic liquid groups and negative charged phosphate groups can interact with the charged groups on the surface of bacteria. Phenylboronic acid can interact with the peptidoglycan and teichoic acid on the surface of bacteria. Certain surface groups and charge property of each kind of bacteria may lead to different binding intensity. Thus, we expected that the common foodborne pathogenic bacteria with phenotypes differences could be reflected from the fluorescence signal. Furthermore, each UCNPs@COPs probe could respond to the various species of bacteria in a unique manner and generate corresponding features fluorescence signal. The three UCNPs@COPs probes could be employed to constitute a three-probe sensor array to realize the identification of bacterial species (Scheme 1).

In an attempt to optimize the working conditions of the three UCNPs@COPs probes, the ubiquitous and well-studied Gram-negative E.coli was chosen as a model bacterium. The physiological saline containing 10\% DMSO was selected as the working media, which could well maintain the dispersion of UCNPs probes and didn't damage the bacterial cell morphology. The effect of the concentrations of UCNPs@COPs probes and incubation time against E.coli were first examined. As shown in Additional file 1: Figures S8 and S9, the relative fluorescence intensities $\left(\left(\mathrm{F}_{\mathrm{i}}-\mathrm{F}_{0}\right) / \mathrm{F}_{0}\right)$ of the three UCNPs@ COPs probes towards bacteria varied with the increase of UCNPs@COPs concentrations and incubation time. Consistently, the fluorescence emission intensities of the three UCNPs@COPs probes could be enhanced by E. coli
$\left(\mathrm{OD}_{600}=0.5\right)$. The strongest fluorescence response was obtained after incubation about $6 \mathrm{~min}$ at $0.20 \mathrm{mg} \mathrm{mL}-1$ of each UCNPs@COPs probe. Therefore, such probe concentration and incubation time were adopted for the following experiments. Meanwhile, the bacterial concentration played an important role. As shown in the Additional file 1: Figure S10, the fluorescence emission of each fluorescence probe enhanced with the increase of E.coli concentration, and the difference among these emissions was the largest at $\mathrm{OD}_{600}=0.5$. Such bacterial concentration $\left(\mathrm{OD}_{600}=0.5\right)$ was preliminary chosen for the following experiments.

In order to further evaluate the discrimination ability of UCNPs@COPs probes, each UCNPs@COPs probe was treated with alive or dead E.coli and S. aureus, respectively, and their fluorescence responses were recorded. As we expected, the fluorescence probes could realize the identification of the alive or dead E.coli and S. aureus. The alive E.coli and S. aureus could enhance the fluorescence of UCNPs@COPs probes more significant than that of the dead one treated with ultrasonic (Fig. 3a, b).

Furthermore, the fluorescence responses of the three UCNPs@COPs probes towards Gram-negative E. coli and Gram-positive $S$. aureus were preliminary investigated. As seen in Fig. 3c, the fluorescence responses of each UCNPs@COPs probe towards E. coli and S. aureus were obvious. Each UCNPs@COPs probe showed different fluorescence responses towards the two bacteria, which was mainly on account of the difference of morphology between E. coli (thallus with blunt ends) and $S$. aureus (small spherical thallus). Meanwhile, all the three UCNPs@COPs probes revealed unique fluorescence response against each kind of bacterium, and the dramatic enhancement could reflect the fairly degree of interaction between UCNPs@COPs probes and bacteria due to the differences in binding ability. Obviously, UCNPs@COPs 1 (UCNPs modified with boronic acid 

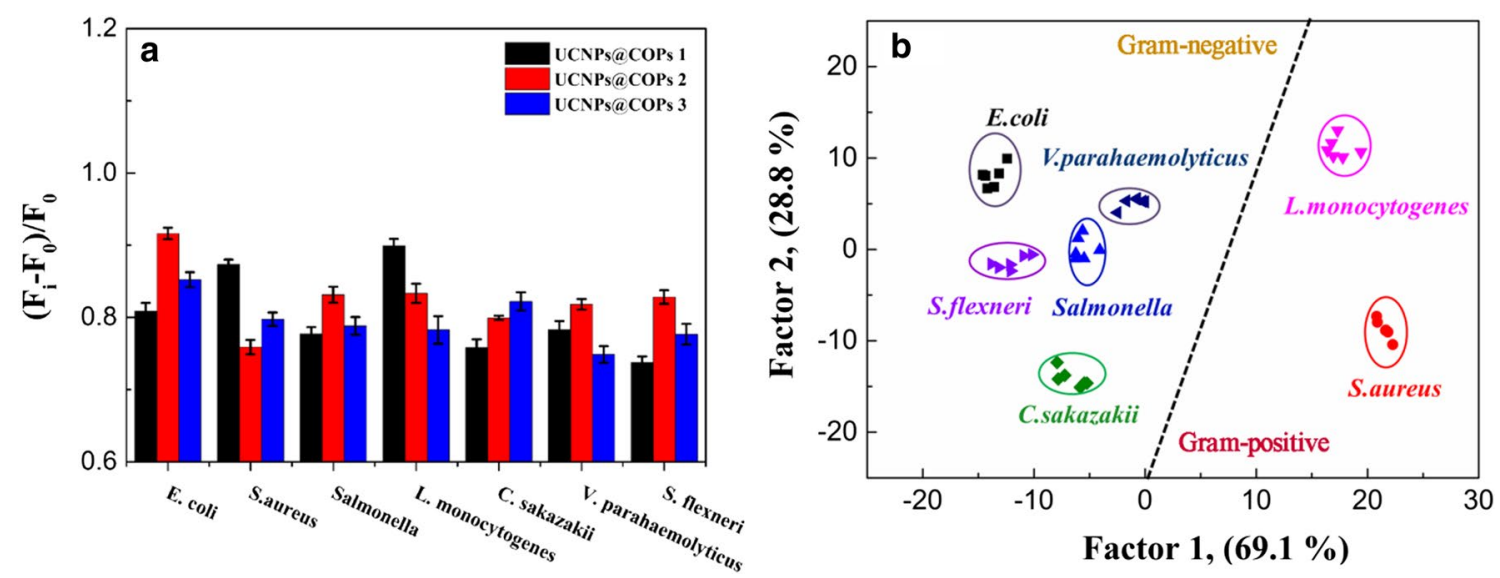

Fig. 4 a Signal patterns of the relative fluorescence intensity variety $\left(\left(F_{i}-F_{0}\right) / F_{0}\right)$ of three kinds of UCNPs@COPs probes in the presence of seven different bacteria $\left(\mathrm{OD}_{600}=0.5\right)$. All values represent the average of six replicates. $\mathbf{b}$ Canonical score plot for the response patterns of bacteria obtained from LDA. Each point represents the response pattern for single bacterial species to the array

groups) displayed the strongest fluorescence emission towards $S$. aureus than that of UCNPs@COPs 2 (UCNPs modified with imidazole ionic liquid). The reasons were attributed to the facts that there were more peptidoglycan and teichoic acid on the surface of Gram-positive bacteria, and boronic acid groups combine strongly with cis-diol molecules. On the other hand, more negative surface charges on the surface of Gram-negative bacteria led to the highest fluorescence emission of UCNPs@COPs 2 towards $E$. coli due to the strong electrostatic interaction between imidazole quaternary ammonium salt and negative charge groups on the surface of $E$. coli. In addition, UCNPs@COPs 3 (UCNPs modified with negative charge phosphate groups) could form weak electronic interaction with the molecules on bacterial cell walls and generate the fluorescence response, however, which was the weakest among these probes. Herein, considering the complexity of bacteria, we concluded that both the spotlight effect of bacterial cell and the interaction between bacteria and the fluorescence probes were the possible sensing mechanisms. The extents of fluorescence response of UCNPs@COPs probes were dependent on the types of bacteria and their multiple surface molecules, which could provide the possibility to distinguish diverse bacteria.

\section{Bacteria detection and discrimination using fluorescent sensor array}

Since UCNPs@COPs probes could generate the different fluorescent response towards diverse bacteria, we further
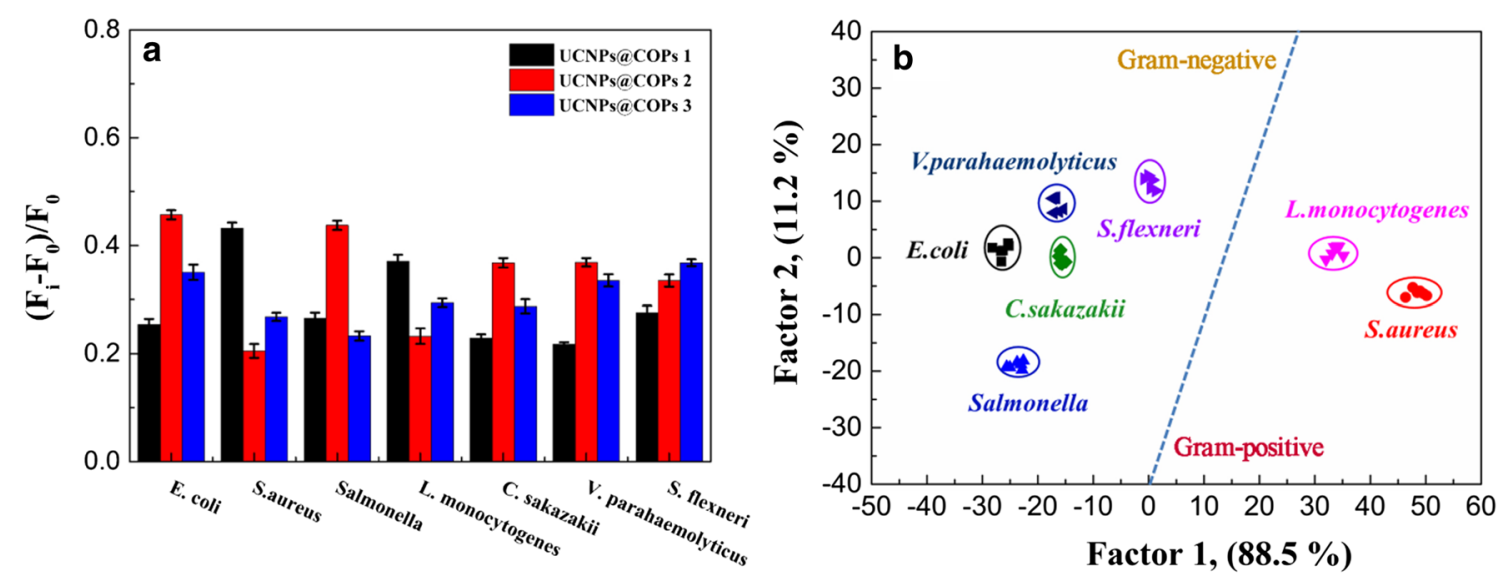

Fig. 5 a Signal patterns of the relative fluorescence intensity variety $\left(\left(F_{i}-F_{0}\right) / F_{0}\right)$ of three kinds of UCNPs@COPs probes in the presence of seven different bacteria $\left(\mathrm{OD}_{600}=0.1\right)$. All values represent the average of six replicates. $\mathbf{b}$ Canonical score plot for the response patterns of bacteria obtained from LDA. Each point represents the response pattern for single bacteria species to the array 


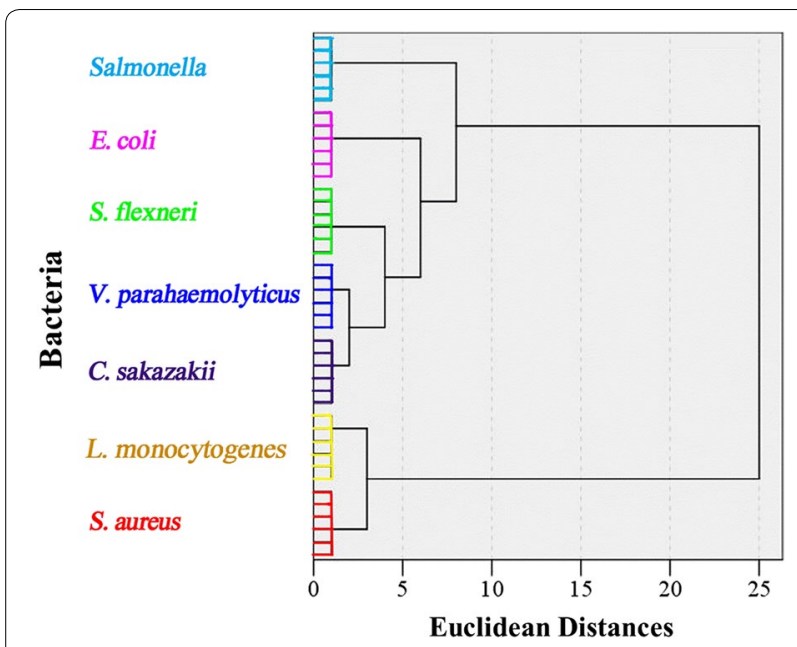

Fig. 6 Dendrogram generated by hierarchical cluster analysis of the seven different bacteria

investigated the fluorescent performances of UCNPs@ COPs probes towards seven kinds of common foodborne pathogenic bacteria (including E. coli, S. aureus, Salmonella, L. monocytogenes, C. sakazakii, S. flexneri, and V. parahaemolyticus). To avoid unnecessary signal fluctuation, the seven kinds of bacteria with a concentration $\left(\mathrm{OD}_{600}=0.5\right)$ were incubated with $0.20 \mathrm{mg} \mathrm{mL}^{-1}$ of three UCNPs@COPs probes in physiological saline containing $10 \%$ DMSO for $6 \mathrm{~min}$, and the physiological saline alone as a control. All tests were performed in six replicates. Then the fluorescence spectra were recorded, and the parameter $\left[\left(\mathrm{F}_{\mathrm{i}}-\mathrm{F}_{0}\right) / \mathrm{F}_{0}\right]$ was further utilized to characterize the fluorescence response patterns of each UCNPs@ COPs-based materials against the seven kinds of bacteria. To our delight, the three probes exhibited significant fluorescence response towards each kind of bacterium (Fig. 4a). The fluorescence response was closely related to the bacterial species, and the achieved fluorescence response could be utilized as the "identification fingerprints" of bacteria for the specific discrimination.

To further maximize the separation ability of the fluorescent sensor array, the fluorescence response patterns of the sensor array were combined, producing a training matrix $(3 \times \mathrm{UCNPs} @ \mathrm{COPs} \times 7$ bacteria $\times 6$ replicates). Then the matrix was classified by linear discriminant analysis (LDA) using the analysis software SPSS (version 22.0), which was a powerful statistical method to separate and identify multiple targets simultaneously by using their linear combination of features. LDA converted the training matrix into canonical scores according to their Mahalanobis distance (Additional file 1: Table S3). Three canonical factors (69.1\%, $28.8 \%$, and $2.1 \%$ ) were generated for the total variance.

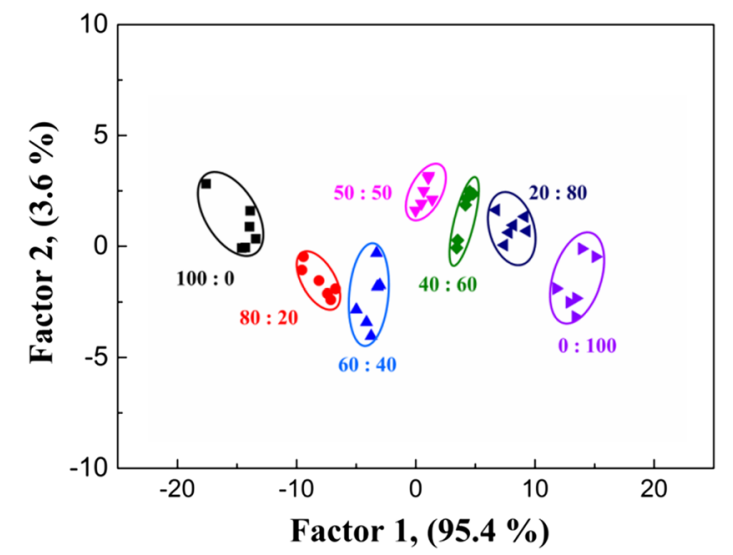

Fig. 7 Canonical score plot for the UCNPs@COPs-based fluorescent sensor array against the bacteria mixtures of E. coli and S. aureus with different ratios (100:0, 80:20, 60:40, 50:50, 40:60, 20:80, and $0: 100)$, respectively in physiological saline. In each case, a bacteria concentration of $\mathrm{OD}_{600}=0.1$ was used. Each point represents the response pattern for single bacterial mixtures to the array

The $2 \mathrm{D}$ canonical scores were plotted according to the first two most important discrimination factors where each point represented the fluorescence response of UCNPs@COPs to each kind of bacteria. We observed that the seven kinds of bacteria were well-clustered without any overlap and discriminated thoroughly from each other (Fig. 4b). The 100\% classification accuracy for each set of bacteria was confirmed according to Jackknife classification (Additional file 1: Table S4), suggesting the extraordinary properties of the threechannel sensor array in discrimination of bacteria. Seven bacteria clustered independently according to their categories in the 2D canonical scores plot, where the Gram-positive bacteria positioned at the right and the Gram-negative bacteria at the left, indicating a certain correlation between bacterial Gram types and the three UCNPs@COPs probes. Furthermore, we simplified the sensor array by removal of one UCNPs@ COPs probe. However, the satisfactory discrimination between seven bacteria was not achieved based on the two-channel UCNPs@COPs sensor array (Additional file 1: Figure S11), indicating that more different UCNPs@COPs probes could distinguish more bacteria.

To validate the efficiency of the fluorescence sensor array, we further decreased the concentration of bacteria to $\mathrm{OD}_{600}=0.1$ (approximates to $10^{6} \mathrm{cfu} \mathrm{mL}^{-1}$ seen in Additional file 1: Table S2). To our delight, the fluorescent sensor array still revealed a good discrimination and accuracy towards the low concentration bacteria (Fig. 5 and Additional file 1: Table S5). Therefore, our fluorescent sensor array displayed better recognition ability and 

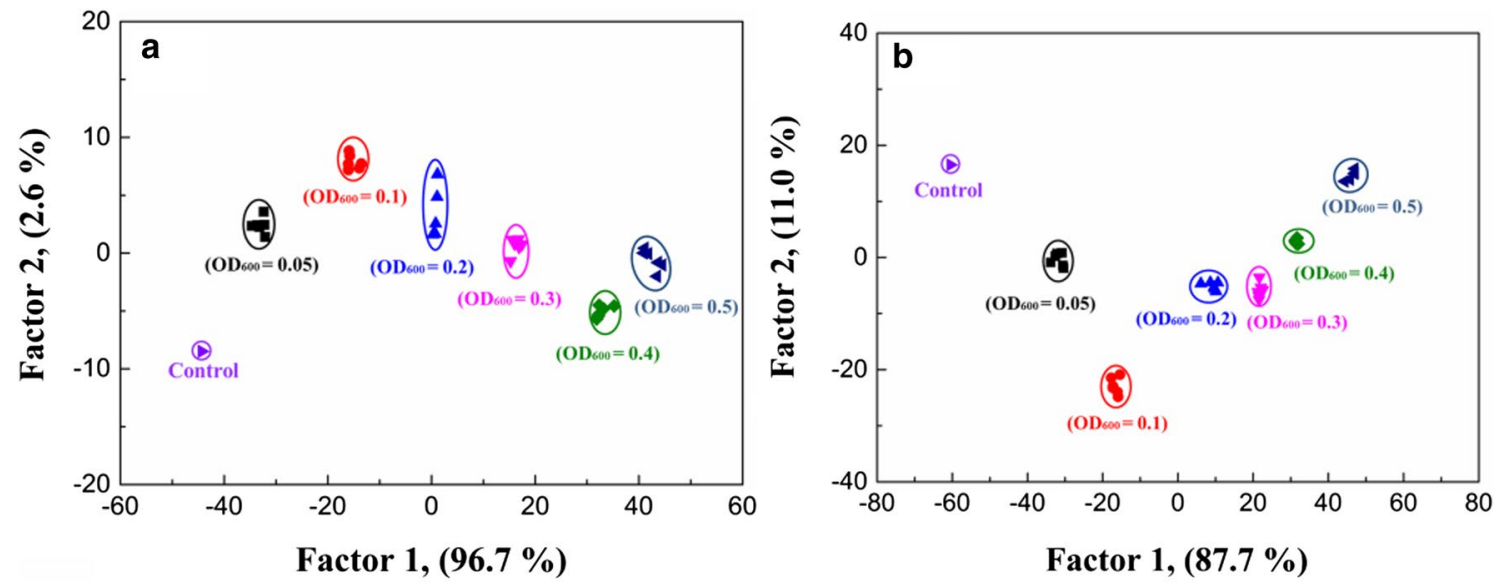

Fig. $82 \mathrm{D}$ canonical score plots obtained with the fluorescent sensor array treated with E.coli (a) and S. aureus (b) at different concentrations $\left(\mathrm{OD}_{600}\right.$ from 0.5 to 0.05 ). Each point represents the response pattern for single bacterial concentrations to the array
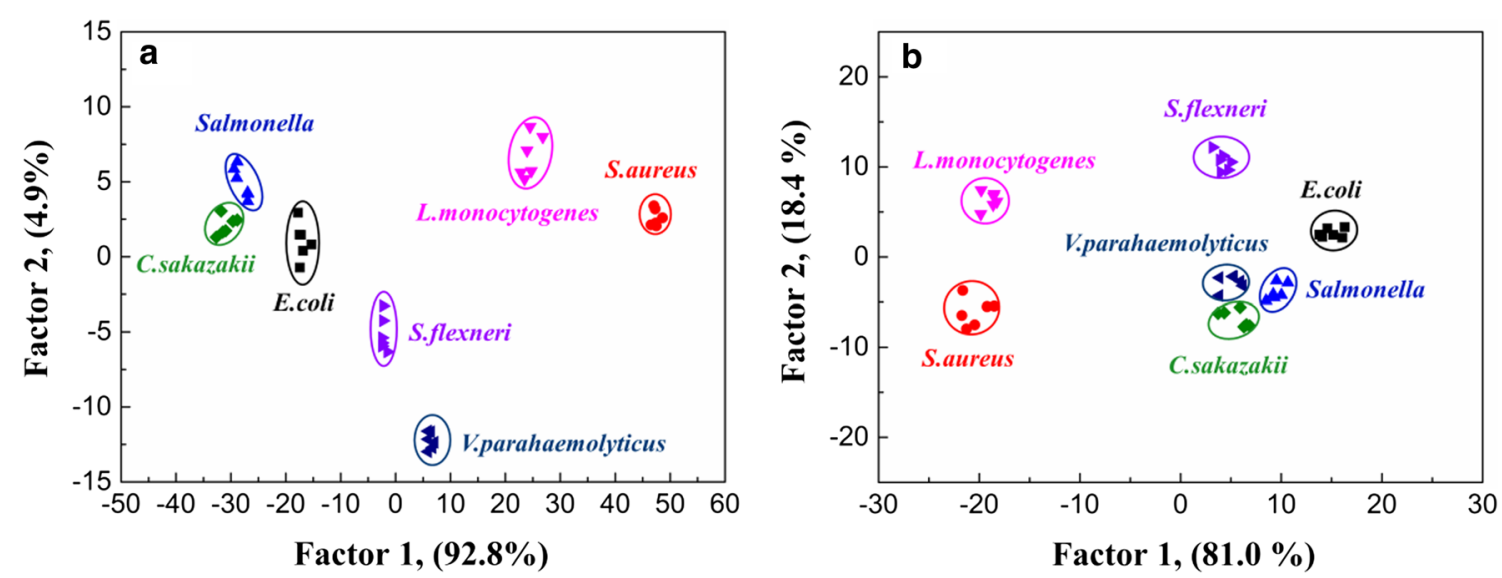

Fig. 9 Discrimination of bacteria in milk and beef culture solution. a LDA plot for the discrimination of bacteria in milk $\left(O D_{600}=0.1\right)$. $\mathbf{b} L D A$ plot for the discrimination of bacteria in beef culture solution $\left(\mathrm{OD}_{600}=0.1\right)$. Each point represents the response pattern for single bacterial species to the array

Table 1 Accuracy (\%) of the blind tests obtained from the fluorescent sensor array

\begin{tabular}{lllll}
\hline Bacteria source & Tap water & Milk & Beef & Total \\
\hline Number of samples & 21 & 21 & 21 & 63 \\
Correctly identified & 21 & 18 & 19 & 58 \\
Accuracy (\%) & $100 \%$ & $85.7 \%$ & $90.5 \%$ & $92.1 \%$ \\
\hline
\end{tabular}

a higher sensitivity $\left(\mathrm{OD}_{600}=0.1\right)$ over the previous report $\left(\mathrm{OD}_{600}=1.0\right)[3,18]$.

In order to further explore the ability of this fluorescent sensor array, HCA was employed to analyse the similarities between the selected bacteria in a step-bystep way according to the Euclidean distances, which was a powerful classify method [18]. The dendrogram produced from HCA based on average linkage method obviously revealed seven distinct clusters, and each cluster represented one kind of bacteria (Fig. 6). Meanwhile, the four Gram-negative bacteria (E. coli, Salmonella, C. sakazakii, V. parahaemolyticus, and S. flexneri) matched at the same level, whereas the two Gram-negative bacteria (S. aureus and L. monocytogenes) at the same level, revealing the more similarity among the bacteria. The results demonstrated that the fluorescent sensor array could discriminate the different Gram states of bacteria.

\section{Discrimination of bacteria mixtures}

The coexisting of foodborne bacteria in the nature environment was not fresh, which make it of great 
significance to distinguish the bacteria mixture. In order to investigate the performance of this fluorescent sensor array towards bacteria mixture, E. coli and $S$. aureus were employed as the model, and the mixtures of two model bacteria with different ratios (100:0, 80:20, 60:40, 50:50, $40: 60,20: 80$, and $0: 100$ ) were design to simulated bacterial coexistence. Then, each mixture with the concentration of $\mathrm{OD}_{600}=0.1$ was identified using the constructed upconversion fluorescent sensor array (Additional file 1: Table S6). The resultant canonical score plot has shown that the different ratios of bacterial mixtures could be successfully separated without any overlapping, which further proved the potential ability of the proposed fluorescent sensor array for discriminating bacteria mixture (Fig. 7).

\section{Quantitative analysis of bacteria}

Since the fluorescence intensities of UCNPs@COPs probes increased with the concentration of bacteria, E.coli and S. aureus were selected to test the quantitative analysis performance of the proposed fluorescent sensor array by using LDA (6 concentrations $\times 3$ UCNPs@ COPs $\times 6$ replicates, $\mathrm{OD}_{600}$ from 0.05 to 0.50 , Additional file 1: Tables S7 and S8). The result showed that all of the six concentrations of E.coli or S. aureus could be discriminated with $100 \%$ accuracy without any overlap, indicating that the proposed fluorescent sensor array could be well employed for the quantitative analysis of bacteria (Fig. 8).

\section{Identification of bacteria in real samples}

In order to verify the performance of the fluorescent sensor array in real food samples, we further simulated bacterial contaminated food (milk and ground beef) to test the discrimination capacity. The proposed fluorescent sensor array was employed to discriminate the common seven foodborne bacteria in these food samples $(3 \times$ UCNPs@COPs $\times 7$ bacteria $\times 6$ replicates $) . T h e$ training results in presence of the food samples matrix interference (milk and beef) showed that the bacteria could be successfully discriminated in complex samples with the same high-accuracy as in physiological saline, indicating that the sensor array equipped with a good capacity of resisting disturbance (Fig. 9, Additional file 1: Tables S9 and S10). Furthermore, 63 unknown bacteria were spiked in tap water, milk and beef culture solution respectively with a bacteria concentration (about $10^{6} \mathrm{cfu} \mathrm{mL}^{-1}$ ), which were randomly picked from the seven bacteria. Detection and identification of unknown bacteria samples were carried out by assigning the fluorescence response pattern of the unknown samples to the corresponding LDA group defined by the training matrix (Additional file 1: Tables S11-S13). The blind tests results showed $92.1 \%$ accuracy of bacterial discrimination and the results were reliable in tap water with $100 \%$ accuracy of discrimination, allowing for higher accuracy in discrimination of bacteria in beef (90.5\%) than that in milk (85.7\%). The results indicated that the proposed fluorescent sensor array based on UCNPs@COPs materials is great potential for practical applications (Table 1). In addition, the discrimination of bacteria mixtures (E. coli and $S$. aureus) with different ratios in real samples was further investigated. The resultant canonical score plot could be successfully separated without any overlapping, which further demonstrated the excellent discriminating capability of the proposed fluorescent sensor array for bacteria mixture in real samples (Additional file 1: Figure S12 and Tables S14-S16).

\section{Conclusion}

A novel upconversion fluorescent sensor array was constructed for discrimination of bacteria based on surface chemistry modified UCNPs materials with boronic acid, quaternary ammonium salt or phosphate groups. The sensing mechanism was mainly depended on the spotlight effect of bacterial cell and interactions between bacteria and the fluorescent probes. The proposed fluorescent sensor array allowed for the quantitative analysis of bacteria and the good discrimination and classification towards bacteria according to the Gram status. In comparison to previous pattern based microbial identification, intact alive microbes instead of microbial lysates were straightly chosen as analytical target, which could shorten preprocessing and realize fast detection and real-time monitoring (Additional file 1: Table S17). Excellent discriminating power in bacteria mixture and real samples (tap water, milk and beef) encouraged us that the sensor array might accelerate the development of the efficient discrimination of complex bacterial samples.

\section{Supplementary information}

Supplementary information accompanies this paper at https://doi. org/10.1186/s12951-020-00596-4.

Additional file 1. Additional figures and tables.

\section{Abbreviations}

UCNPS: Upconversion nanoparticles; COPs: Co-polymers; TEOS: Tetraethyl orthosilicate; ATS: Allyltriethoxysilane; AIBN: Azodiisobutyronitrile; DMSO: Dimethyl sulfoxide; PBS: Phosphate buffer saline; TEM: Transmission electron microscopy; EDS: Energy-dispersive X-ray spectroscope; FT-IR: Fourier transform infrared spectra; TGA: Thermogravimetric analysis; LDA: Linear discriminant analysis.

\section{Acknowledgements}

The authors are grateful for the financial support provided by the Ministry of Science and Technology of China (project no. 2016YFD0401101) and the National Natural Science Foundation of China (project no. 21375094). 


\section{Authors' contributions}

MY and QD conceived and designed the experiments. MY performed the experiments. MY wrote the paper with support from QD. All authors contributed to the general discussion. All authors read and approved the final manuscript.

\section{Availability of data and materials}

All data generated or analyzed during this study are included in the article and additional file. The additional file is available. Synthesis process of UCNPs, IL-Br, and Co-polymers. EDS, XRD patterns, FITR spectrum, Thermogravimetric analysis, Zeta potential, and Fluorescence emission spectra of UCNPs@COPs materials (Additional file 1: Figures S1-S7). Optimization of assay conditions of the fluorescent sensor array (Additional file 1: Figures S8-S11). Linear discriminant analysis using the sensor array against the bacteria mixtures in real samples (Figure S11). Synthesis of co-polymers (Additional file 1: Table S1). Numbers of bacteria using plate count (Additional file 1: Table S2). Fluorescence response pattern and linear discriminant analysis (Additional file 1: Tables S3-S10 and S14-S16) Detection and identification of unknown bacteria samples (Additional file 1: Tables S11-S13). Comparison of different detection methods (Additional file 1: Table S17)

\section{Consent for publication}

All authors have provided consent for the manuscript to be published.

\section{Competing interests}

The authors declare no competing financial interest.

\section{Author details}

${ }^{1}$ State Key Laboratory of Food Nutrition and Safety, School of Food Engineering and Biotechnology, College of Chemical Engineering and Materials Science, Tianjin University of Science and Technology, Tianjin 300457, People's Republic of China. ${ }^{2}$ Tianjin Key Laboratory of Food Science and Health, School of Medicine, Nankai University, Tianjin 300071, People's Republic of China.

\section{Received: 31 October 2019 Accepted: 20 February 2020}

Published online: 28 February 2020

\section{References}

1. Yin M, Yang B, Wu Y, Wang L, Wu H, Zhang T, Tuohetaribayi G. Prevalence and characterization of Salmonella enterica serovar in retail meats in market place in Uighur, Xinjiang. China Food Control. 2016;64:165-72.

2. Liu GJ, Tian SN, Li CY, Xing GW, Zhou L. Aggregation-induced-emission materials with different electric charges as an artificial tongue: design, construction, and assembly with various pathogenic bacteria for effective bacterial imaging and discrimination. ACS Appl Mater Inter. 2017;9:28331-8.

3. Shen J, Hu R, Zhou T, Wang Z, Zhang Y, Li S, Gui C, Jiang M, Qin A, Tang BZ. Fluorescent sensor array for highly efficient microbial lysate identification through competitive interactions. ACS Sensors. 2018;3:2218-22.

4. Han J, Cheng H, Wang B, Braun MS, Fan X, Bender M, Huang W, Domhan C, Mier W, Lindner T. A polymer/peptide complex-based sensor array that discriminates bacteria in urine. Angew Chem. 2017;56:15246-51.

5. Mocan T, Matea CT, Pop T, Mosteanu O, Buzoianu AD, Puia C, lancu C, Mocan L. Development of nanoparticle-based optical sensors for pathogenic bacterial detection. J Nanobiotechnol. 2017:15:25

6. Zhou C, Xu W, Zhang P, Jiang M, Chen Y, Kwok RTK, Lee MMS, Shan G, Qi R, Zhou X. Engineering sensor arrays using aggregation-induced emission luminogens for pathogen identification. Adv Funct Mater. 2019;29:1805986.

7. Kant K, Shahbazi M-A, Dave VP, Ngo TA, Chidambara VA, Than LQ, Bang DD, Wolff A. Microfluidic devices for sample preparation and rapid detection of foodborne pathogens. Biotechnol Adv. 2018;36:1003-244.

8. Li B, Li X, Dong Y, Wang B, Li D, Shi Y, Wu Y. Colorimetric sensor array based on gold nanoparticles with diverse surface charges for microorganisms identification. Anal Chem. 2017;89:10639-43.

9. Li YC, Xin HB, Lei HX, Liu LL, Li YZ, Zhang Y, Li BJ. Manipulation and detection of single nanoparticles and biomolecules by a photonic nanojet. Light-Sci Appl. 2016;5:e16176.
10. Li Y, Liu X, Yang X, Lei H, Zhang Y, Li B. Enhancing upconversion fluorescence with a natural bio-microlens. ACS Nano. 2017;11:10672-80.

11. Xin H, Li Y, Xu D, Zhang Y, Chen CH, Li B. Single upconversion nanoparticle-bacterium cotrapping for single-bacterium labeling and analysis. Small. 2017;13(14):1603418.

12. Yin M, Wu C, Li H, Jia Z, Deng Q, Wang S, Zhang Y. Simultaneous sensing of seven pathogenic bacteria by guanidine-functionalized upconversion fluorescent nanoparticles. ACS Omega. 2019;4(5):8953-9.

13. Velusamy V, Arshak K, Korostynska O, Oliwa K, Adley C. An overview of foodborne pathogen detection: in the perspective of biosensors. Biotechnol Adv. 2010;28(2):232-54

14. Lee JS, Cho AN, Jin Y, Kim J, Kim S, Cho SW. Bio-artificial tongue with tongue extracellular matrix and primary taste cells. Biomaterials. 2018;151:24-37.

15. Tian XW, Li Z, Pang YX, Li DY, Yang XB. Benzoyl peroxide detection in real samples and zebrafish imaging by a designed near-infrared fluorescent probe. J Agr Food Chem. 2017;65(43):9553-8.

16. Yang $H$, Jie $X$, Wang $L$, Zhang $Y$, Wang $M$, Wei W. An array consisting of glycosylated quantum dots conjugated to MoS2 nanosheets for fluorometric identification and quantitation of lectins and bacteria. Mikrochim Acta. 2018;185(11):512.

17. Li Z, Askim JR, Suslick KS. The optoelectronic nose: colorimetric and fluorometric sensor arrays. Chem Rev. 2019;119(1):231-92.

18. Zheng L, Qi P, Zhang D. Identification of bacteria by a fluorescence sensor array based on three kinds of receptors functionalized carbon dots. Sensor Actuat B Chem. 2019:286:206-13.

19. Ceto X, Voelcker NH, Prieto-Simon B. Bioelectronic tongues: new trends and applications in water and food analysis. Biosens Bioelectron. 2016;79:608-26.

20. Yin M, Zhang C, Li J, Li H, Deng Q, Wang S. Highly sensitive detection of benzoyl peroxide based on organoboron fluorescent conjugated polymers. Polymers. 2019;11(10):1655.

21. Jin B, Wang S, Lin M, Jin Y, Zhang S, Cui X, Gong Y, Li A, Xu F, Lu TJ. Upconversion nanoparticles based FRET aptasensor for rapid and ultrasenstive bacteria detection. Biosens Bioelectron. 2017;90:525-33.

22. Wang K, Li Y, Li H, Yin M, Liu H, Deng Q, Wang S. Upconversion fluorescent nanoparticles based-sensor array for discrimination of the same variety red grape wines. RSC Adv. 2019;9(13):7349-55.

23. Fan D, Wang E, Dong S. Upconversion-chameleon-driven DNA computing: the DNA-unlocked inner-filter-effect (DU-IFE) for operating a multicolor upconversion luminescent DNA logic library and its biosensing application. Mater Horizons. 2019;6(2):375-84.

24. Han Y, An Y, Jia G, Wang X, He C, Ding Y, Tang Q. Facile assembly of upconversion nanoparticle-based micelles for active targeted dual-mode imaging in pancreatic cancer. J Nanobiotechnol. 2018;16(1):7.

25. Zhang Z, Shikha S, Liu J, Zhang J, Mei Q, Zhang Y. Upconversion nanoprobes: recent advances in sensing applications. Anal Chem. 2019:91(1):548-68.

26. Wu S, Ning Y, Chang J, Zhang S. Upconversion photoluminescence enhancement and modulation of NaYF4:Yb, Er through using different ligands. J Lumin. 2013;143:492-7.

27. Recalde I, Estebanez N, Frances-Soriano L, Liras M, Gonzalez-Bejar M, Perez-Prieto J. Upconversion nanoparticles with a strong acid-resistant capping. Nanoscale. 2016;8(14):7588-94

28. Hu P, Wu X, Hu S, Tang Z, Dai G, Liu Y. Upconversion nanoparticle arrays for detecting glycated hemoglobin with high sensitivity and good reusability. RSC Adv. 2016;6(104):102226-30.

29. Yliharsila M, Valta T, Karp M, Hattara L, Harju E, Holsa J, Saviranta P, Waris M, Soukka T. Oligonucleotide array-in-well platform for detection and genotyping human adenoviruses by utilizing upconverting phosphor label technology. Anal Chem. 2011;83(4):1456-61.

30. Yan $Q$, Ding $X Y$, Chen ZH, Xue SF, Han XY, Lin ZY, Yang M, Shi G, Zhang M. $\mathrm{pH}$-Regulated optical performances in organic/inorganic hybrid: a dualmode sensor array for pattern-recognition-based biosensing. Anal Chem. 2018;90(17):10536-42.

31. Wiesholler LM, Genslein C, Schroter A, Hirsch T. Plasmonic enhancement of NIR to UV upconversion by a nanoengineered interface consisting of NaYF4:Yb, Tm nanoparticles and a gold nanotriangle array for optical detection of vitamin B12 in serum. Anal Chem. 2018;90(24):14247-544.

32. Pakkila H, Yliharsila M, Lahtinen S, Hattara L, Salminen N, Arppe R, Lastusaari M, Saviranta P, Soukka T. Quantitative multianalyte microarray 
immunoassay utilizing upconverting phosphor technology. Anal Chem. 2012;84(20):8628-34.

33. Kale V, Pakkila H, Vainio J, Ahomaa A, Sirkka N, Lyytikainen A, Talha SM, Kutsaya A, Waris M, Julkunen I. Spectrally and spatially multiplexed serological array-in-well assay utilizing two-color upconversion luminescence imaging. Anal Chem. 2016;88(8):4470-7.

34. Pan W, Zhao J, Chen Q. Fabricating Upconversion fluorescent probes for rapidly sensing foodborne pathogens. J Agr Food Chem. 2015;63(36):8068-74.

35. Wu S, Duan N, Shi Z, Fang C, Wang Z. Simultaneous aptasensor for multiplex pathogenic bacteria detection based on multicolor upconversion nanoparticles labels. Anal Chem. 2014;86(6):3100-7.

36. Zhang B, Li H, Pan W, Chen Q, Ouyang Q, Zhao J. Dual-color upconversion nanoparticles (UCNPs)-based fluorescent immunoassay probes for sensitive sensing foodborne pathogens. Food Anal Method. 2016;10(6):2036-45.
37. GB 5749-2006. The sanitary standards for drinking water quality of the state standard of the People's Republic of China. http://down.foodmate. net/standard/sort/3/10968.html.

38. Li H, Wang L. NaYF4:Yb ${ }^{3+} / \mathrm{Er}^{3+}$ nanoparticle-based upconversion luminescence resonance energy transfer sensor for mercury (II) quantification. Analyst. 2013;138(5):1589-95.

\section{Publisher's Note}

Springer Nature remains neutral with regard to jurisdictional claims in published maps and institutional affiliations.
Ready to submit your research? Choose BMC and benefit from:

- fast, convenient online submission

- thorough peer review by experienced researchers in your field

- rapid publication on acceptance

- support for research data, including large and complex data types

- gold Open Access which fosters wider collaboration and increased citations

- maximum visibility for your research: over 100M website views per year

At BMC, research is always in progress.

Learn more biomedcentral.com/submissions 